\title{
THE ROLE OF VIRTUAL NOISE IN UNCONSTRAINED FREQUENCY DOMAIN EQUALIZATION
}

\author{
M. Magarini, A. Spalvieri \\ Dipartimento di Elettronica e Informazione, Politecnico di Milano, \\ Piazza Leonardo da Vinci, 32, I-20133 Milano (Italy), \\ \{magarini,spalvier\}@elet.polimi.it
}

\begin{abstract}
Decision feedback equalization is a popular method for signal detection that allows a good trade off between complexity and performance. The complexity of the Decision Feedback Equalizer (DFE) is mainly concentrated in the feedforward filter, which is often realized in FIR form. When the number of taps of the FIR filter needed to obtain nearly optimal performance becomes large, one may take advantage of the efficiency of the FFT/IFFT algorithm, realizing the feedforward filter in the discrete frequency domain. In this paper we consider the Minimum Mean Square Error (MMSE) DFE with feedforward filter in the discrete frequency domain, assuming perfect knowledge of a static channel. We move from the observation that the optimal MMSEDFE feedforward filter is IIR, and point out that the classical scheme of unconstrained frequency domain filtering with overlap/save digital signal processing may induce substantial increase in the error rate when the approximation of the linear convolution between the received signal and the mentioned IIR to a circular convolution is poor. Our main finding is that the error rate can be significantly improved by deliberately augmenting the power of the noise in the computation of the transfer function of the frequency domain feedforward filter.
\end{abstract}

Keywords - Frequency domain equalization, equalization, intersymbol interference.

\section{INTRODUCTION}

Decision feedback equalization is a simple and effective technique that is often adopted in detection of signals affected by noise and multipath. The Decision Feedback Equalizer (DFE) consists of a FeedForward Filter $(\mathrm{FFF})$, an instantaneous detector, and a FeedBack Filter $(\mathrm{FBF})$ that operates cancellation of the InterSymbol Interference (ISI) due to the past data by feeding back a weighted combination of the past decisions taken by the detector. The most popular DFE dates back to Monsen [1]. In the DFE due to Monsen, which is known as the Minimum Mean Square Error DFE (MMSE-DFE), the FFF and the FBF optimize the mean square error between the input to the detector and the transmitted symbol.

The complexity of the MMSE-DFE is mainly concentrated in the FFF. Although the optimal FFF of the
MMSE-DFE is IIR, the designer often prefers to implement it in the FIR form. In this case, often nearly optimal performance is obtained only at the cost of a large number of taps. Of course, the large number of taps brings the cost of the large number of hot multiplications made by the FIR during the processing of the received signal. Furthermore, the designer has to face the problem of optimizing the FIR. Many methods have been proposed in the past to efficiently compute the weights of an optimized FIR, e.g. [2]. However, all these methods have a complexity that goes with $N^{2}$, where $N$ is the number of taps of the FIR. Therefore, when the number of taps that are needed to obtain nearly optimal performance becomes too large, the designer is led to consider the implementation of the FFF in the discrete frequency domain, taking two advantages. The first one is the well-known efficiency of the FFT/IFFT algorithm for $N \geq 32$. The second one is the efficiency in the computation of the transfer function of the filter that can be achieved by exploiting the approach of Belfiore and Park [3].

Together with the two mentioned advantages, the discrete frequency domain FFF brings also one problem. Basically, the problem with the discrete frequency domain approach is that the discrete frequency domain filter performs the circular convolution, while the channel performs the linear convolution. In this regard, one can take one out of these two approaches: either to force the channel to perform circular convolution or to force the receive filter to perform linear convolution. Recently Falconer et al. [4] and Benvenuto and Tomasin [5] have proposed to adopt frequency domain equalization in conjunction with the cyclic extension technique, where the first of the two mentioned approaches is exploited. In the cyclic extension technique, at the transmit side the data sequence is organized into blocks, and at the end of each block a guard interval is placed where the initial part of the data sequence of that block is attached. At the receive side the cyclic extension is discarded before the FFT. If the duration of the guard interval is greater than or equal to the duration of the impulse response of the channel, the channel operates on the data block the circular convolution. As a consequence, the cascade of channel and receive filter operates on the data block 
the circular convolution, and the cyclic impulse response resulting from the cascade of channel and receive filter can be optimized (e.g., in the MMSE sense) for the given number of FFT/IFFT points. The drawback of the cyclic extension technique is that the channel is occupied for part of the time by the cyclic extension. Transmission of the cyclic extension can be avoided by resorting to digital signal processing techniques such as the overlap/save technique [6], which is the first step towards forcing the receive filter to approximate the linear convolution. According to the overlap/save technique, at the receive side one takes successive blocks of $N$ samples in time domain such that the $n$-th block overlaps the $(n-1)$-th block in a prescribed number of samples, which should be not lower than the duration of the impulse response of the channel. In what follows we assume the overlap to be $N / 2$. The technique proceeds by performing frequency domain filtering on $N$ points, and by discarding $N / 2$ samples after the IFFT. Pioneering work was made in this direction by Ferrara [7] and by Mansour and Gray [8] in the context of adaptive linear MMSE equalization. Specifically, Ferrara observed that linear convolution is obtained by the overlap/save technique only if the duration of the impulse response of the receive filter is not greater than $N / 2$. To overcome this problem, Ferrara proposed to force $N / 2$ zeros in the tail of the impulse response of the filter. However, in the frequency domain approach what is optimized and used is the transfer function of the filter. Hence the zeros can be forced only at the cost of FFT plus IFFT, the FFT to obtain the impulse response from the transfer function, the IFFT to come back to the frequency domain from the impulse response. Mansour and Gray observed that the FFT and the IFFT can be often skipped because good MSE can be obtained also by the unconstrained frequency domain equalizer, where no zeros are forced. Later, Feuer and Cristi demonstrated in [9] that the unconstrained frequency domain equalizer achieves the minimum average MSE for the given number of points of the FFT/IFFT, where the average is made on the block of $N / 2$ samples after the discard. However, Feuer and Cristi did not examine the performance of the equalizer in the context of data transmission, therefore they did not consider the impact of nonlinear convolution on the error rate.

The paper is organized as follows. In section II the MMSE-DFE is described. In section III the analysis of the error performance of the MMSE-DFE with unconstrained frequency domain FFF is given, and the use of virtual noise is proposed. In section IV simulation results that enlighten the merits of our proposal are presented. In section $\mathrm{V}$ conclusions are drawn.

\section{UnCONSTRAined FreqUenCy DOMAin FEEDFORWARD FILTER FOR THE MMSE-DFE}

Transmission of i.i.d. binary antipodal symbols taken from $\{+1,-1\}$ over a time-discrete baseband linear sys- tem corrupted by zero-mean Additive White Gaussian Noise (AWGN) is considered. Extension to nonbinary symbols as well as to passband transmission is possible, but it is not pursued here. The receiver that we consider consists of the matched filter and of the MMSE-DFE. In what follows, we adopt the $z$-transform to represent sequences. For example, the $z$-transform of the transmitted sequence $\left\{a_{k}\right\}$ is

$$
a(z)=\sum_{k} a_{k} z^{-k}
$$

Let

$$
r(z)=\sigma^{2}+\sum_{k} z^{-k} \sum_{i} g_{i} g_{i+k}
$$

be the $z$-transform of the autocorrelation of the observed signal, where $\sigma^{2}$ is the power of the noise, and the sequence $\left\{g_{k}\right\}$ is the impulse response of the linear system. The Signal-to-Noise Ratio (SNR) is

$$
\mathrm{SNR}=\frac{r_{0}-\sigma^{2}}{\sigma^{2}} .
$$

Following the classical approach of Monsen [1], the FBF of the MMSE-DFE is obtained from the spectral factorization

$$
\alpha d(z) d\left(z^{-1}\right)=r(z)
$$

where $\alpha$ is a scalar, and the $d(z)$ that is monic, causal, and minimum phase is taken. The scalar $\alpha$ can be calculated as

$$
\log (\alpha)=\frac{1}{2 \pi} \int_{-\pi}^{\pi} \log \left(r\left(e^{j \omega}\right)\right) d \omega
$$

(where the logarithms can have any common base). Note that (1) does not require the knowledge of $d(z)$. The minimum phase property guarantees that the energy is concentrated in the first taps of the impulse response, which is a desirable property in a DFE scheme. The MMSE-DFE FFF is

$$
p(z)=\frac{d(z)}{r(z)}=\frac{1}{\alpha d\left(z^{-1}\right)} .
$$

Equation (2) shows that $p(z)$ is IIR when $r(z)$ is FIR. Note that $\sigma^{2}>0$ guarantees the existence of both $d(z)$ and $p(z)$. Let $y(z)$ be the $z$-transform of the sequence at the output of the matched filter, and let

$$
e(z)=y(z) p(z)-d(z) a(z)
$$

be the $z$-transform of the error sequence. The error sequence can be seen as the sum of noise and ISI:

$$
e(z)=n(z) p(z)-\sigma^{2} a(z) p(z)
$$

where $n(z)$ is the noise sequence at the output of the matched filter. After straightforward manipulation, for 
the $z$-transform of the autocorrelation of the error sequence one finds

$$
E\left\{e(z) e\left(z^{-1}\right)\right\}=\frac{\sigma^{2}}{\alpha} .
$$

Equation (3) is a classical result in the literature of DFE. It shows that the MMSE is $\sigma^{2} / \alpha$ and that the error sequence is white.

Computation of the feedback filter can be performed as follows. Let $r(z)$ be invertible, and let

$$
s(z)=r^{-1}(z) .
$$

The coefficients of $s(z)$ are the inverse Fourier transform of the spectrum $r^{-1}\left(e^{j \omega}\right)$ :

$$
s_{k}=\frac{1}{2 \pi} \int_{-\pi}^{\pi} \frac{e^{j \omega k}}{r\left(e^{j \omega}\right)} d \omega .
$$

Let $r_{i}=0$ for $|i|>\nu$, and let $\mathbf{d}_{\nu}=\left(-d_{1},-d_{2}, \ldots,-d_{\nu}\right)$. Also, let $\mathbf{s}_{\nu}=\left(s_{1}, s_{2}, \ldots, s_{\nu}\right)$, and let $\mathcal{S}_{\nu}$ be the $\nu \times \nu$ Toeplitz matrix constructed from $s(z)$, so that the element $s_{i, j}$ of $\mathcal{S}_{\nu}$ is $s_{|i-j|}$. After Belfiore and Park [3], we know that $\mathbf{d}_{\nu}$ can be computed by solving the linear system

$$
\mathbf{d}_{\nu} \mathcal{S}_{\nu}=\mathbf{s}_{\nu}
$$

Equation (4) is a linear system of $\nu$ equations known as the Yule-Walker equations. Equation (4) can be efficiently solved via the Levinson-Durbin algorithm, which requires $\nu^{2}+\mathcal{O}(\nu)$ operations.

Efficient implementation of the MMSE-DFE can be worked out by realizing the FFF in the discrete frequency domain and the FBF in the time domain. Efficient computation of the coefficients of the FFF and of the FBF can be performed by translating the approach of Belfiore and Park from the frequency domain to the discrete frequency domain. Specifically, one can compute the samples of $s\left(e^{j \omega}\right)$ in the discrete frequency domain and take the IFFT. The result is an approximation to the sequence $\left\{s_{k}\right\}$. The first $\nu+1$ terms of the mentioned approximation are used in (4) to obtain an approximation to the FBF, which, after FFT, can be used in (2) to obtain the FFF in the discrete frequency domain. Note that one obtains an approximation to the optimal FFF because, since the optimal FFF is IIR, sampling in frequency domain causes aliasing in time domain.

Computation of the FFF and FBF in the discrete frequency domain has been recently rediscovered and proposed by Benvenuto and Tomasin in [5].

Finally, we mention that, to obtain the experimental results to be presented in section IV, we have realized the cascade of matched filter and FFF in the discrete frequency domain. This is done by computing the FFF in the discrete frequency domain as described above, and by multiplying it by the FFT of the zero-padded impulse response of the matched filter.

\section{Error Performance of the MMSE-DFE With UnCONSTRAined Frequency Domain FFF}

The key observation in performance evaluation of the MMSE-DFE with unconstrained frequency domain FFF is that the channel operates linear convolution while the receive FFF operates circular convolution. The result is that the cascade of channel and FFF should be modelled as a linear time-variant system. Assume FFT/IFFT on $N$ points and overlap/save with overlap over $N / 2$ samples. Assume also that the impulse response of the channel is FIR with $\nu+1$ samples, $\nu \leq N / 2$, and that the impulse response of the FFF is unconstrained, which means that its duration is $N$. With these assumptions, the linear time-variant system formed by the cascade of channel and receive FFF is characterized by $N / 2$ impulse responses, the $i$-th of which is the impulse response of the system when the impulse is applied at time $i$, $i=0,1, \cdots, N / 2-1$. Note that while in the cyclic extension technique one has a time-variant linear system where the impulse response at time $i$ is obtained by cyclically shifting of $i$ samples the impulse response at time 0 , here the $N / 2$ impulse responses do not have this regularity.

To describe the set of impulse responses we need to introduce some notation. Let

$$
[u(z)]_{i}^{j}=\sum_{k=i}^{j} u_{k} z^{-k},
$$

and let $\odot_{N}$ be the "N-cyclic product" between $z$ transforms:

$$
u(z) \odot_{N} b(z)=\sum_{k} z^{-k N} u(z) b(z) .
$$

Let $c(z)$ be the $z$-transform of the IFFT of the discrete frequency domain filter that includes matched filter and FFF filter, and let $h_{i}(z)$ be the $z$-transform of the impulse response of the time-variant system from the data source to the output of the FFF when the impulse is applied at time $i$. With the assumptions we have made, the samples of $h_{i}(z)$ may occupy up to three FFT/IFFT blocks. Assume $0 \leq i \leq N / 2-1$, that the first block occupied by $h_{i}(z)$ starts at time $-N / 2$ and terminates at time $N / 2-1$, and that, according to [6], the first $N / 2$ samples after the IFFT are discarded. With these assumptions $h_{i}(z)$ is

$$
\begin{aligned}
z^{-i} h_{i}(z)= & {\left[\left[z^{-i} g(z)\right]_{0}^{N / 2-1} \odot_{N} c(z)\right]_{0}^{N / 2-1} } \\
& +\left[\left[z^{-i} g(z)\right]_{0}^{N-1} \odot_{N} c(z)\right]_{N / 2}^{N-1} \\
& +\left[\left[z^{-i} g(z)\right]_{N / 2}^{3 N / 2-1} \odot_{N} c(z)\right]_{N}^{3 N / 2-1}, \\
& h_{i}(z)=h_{i+N / 2}(z) .
\end{aligned}
$$

The sequence at the output of the FFF is the sum of noise and of the superimposition of the above family of impulse 
responses, each member of the family being multiplied by the proper binary symbol

$$
x(z)=m(z)+\sum_{i} a_{i} h_{i}(z) z^{-i},
$$

where $m(z)$ is the noise sequence. The $z$-transform of the error sequence turns out to be

$$
e(z)=x(z)-d(z) a(z)
$$

The error sequence can be seen as the sum of noise and ISI, however, while the noise is stationary, the ISI is not. More precisely, let $h_{j, i}$ be the amplitude of the $j$-th impulse response at time $i$. The coefficient of the interference caused by the $j$-th symbol on the $i$-th symbol is

$$
v_{i, j}=h_{j, i}-d_{i-j}
$$

The set of coefficients of the ISI at time $i$ is obtained by fixing $i$ and taking the set $\left\{v_{i, j}\right\}$. The noise sequence $\left\{m_{k}\right\}$ is a stationary sequence of zero mean Gaussian random variables with variance

$$
\sigma_{m}^{2}=\sigma^{2}\left[c(z) c\left(z^{-1}\right)\right]_{0} .
$$

From the above theory one can derive a family of $N / 2$ probability density functions of ISI plus noise, and use them in the evaluation of the error performance. Unfortunately, error propagation, which may take place in the DFE, complicates the analysis. To overcome this difficulty we evaluate by analysis only the First Error Event Rate (FEER), which is the error probability given correct decisions in the memory of the FBF, and resort to computer simulation to measure the Bit Error Rate (BER). The FEER has been evaluated by applying the method of Vanelli and Shehadeh [10] to each of the $N / 2$ probability density functions, and then taking the average.

As it will be clear from the experimental results, the time-variant nature of the system may lead to severe performance degradation, because some of the $N / 2$ time instants are severely affected by the detrimental effects of the circular convolution. To mitigate this effect, one can deliberately augment the power of the noise in the spectral factorization,

$$
\alpha d(z) d\left(z^{-1}\right)=r(z)+\lambda, \quad \lambda \geq 0
$$

and then use the $d(z)$ and the $p(z)$ that come from (5). The effect of $\lambda>0$ is that of diminishing the effective duration of the IIR impulse response of the optimal FFF, mitigating in this way the detrimental influence of both time domain aliasing and circular convolution.

It is worth observing that the countermeasure that we propose has found so far widespread application in mitigating various detrimental phenomena that may affect the equalizer. More precisely, Gitlin et al. proposed in

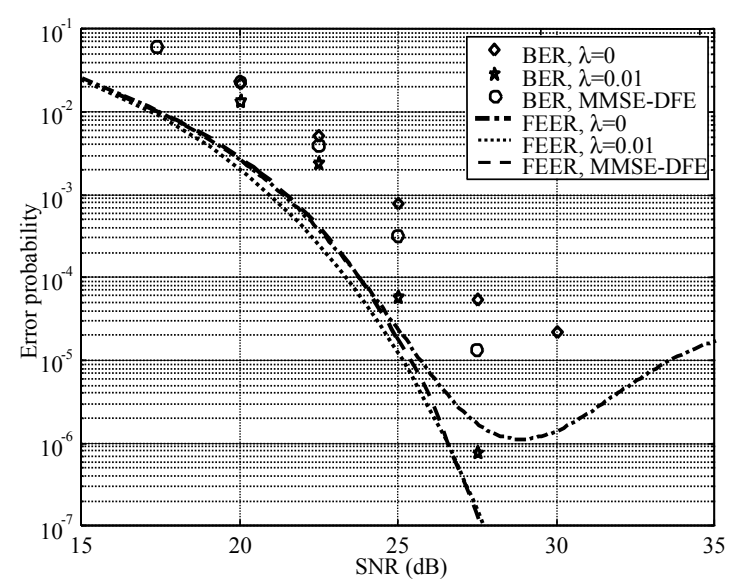

Fig. 1. Computed FEER for the optimal MMSE-DFE and for $N=64$ with $\lambda=0$ and $\lambda=0.01$. Simulated BER for the optimal MMSE-DFE and for $N=64$ with $\lambda=0$ and $\lambda=0.01$.

[11] the use of virtual noise in the context of MMSE adaptive fractionally spaced linear equalization to improve the stability of the hill-conditioned linear equalizer. Later, Magarini et al. observed that the same countermeasure can be adopted with the blind equalizer [12]. Berdnaz and Cioffi [13] proposed the use of virtual noise to mitigate error propagation in the DFE, and Magarini and Spalvieri [14] pointed out the benefits of virtual noise in equalization based on an unperfect channel estimate.

\section{Experimental Results}

To show the benefits of our proposal we adopt the time discrete channel with $\nu=6$ studied in [15]. The impulse response of this channel is $g(z)=0.176+0.316 z^{-1}+$ $0.476 z^{-2}+0.532 z^{-3}+0.476 z^{-4}+0.316 z^{-5}+0.176 z^{-6}$. Note that the channel has three zeros on the unit circle. The frequency domain filtering is performed on $N=64$ points. Fig. 1 reports the computed FEER and the simulated BER versus SNR for $\lambda=0$ and $\lambda=0.01$. The BER is measured by a random sequence of $10^{7}$ binary symbols. The simulated FEER is not shown in the figure since it is virtually equal to the computed one. The performance of the optimal MMSE-DFE is reported as reference. The introduction of virtual noise improves both the BER and the FEER. While the benefits on the BER are predicted in [13], our results demonstrate that a judicious design of $\lambda$ slightly improves also the FEER. Note that the performance obtained by adding virtual noise is better than the one of the optimal MMSE-DFE. This result can be explained by considering that the minimization of the MSE does not guarantee the best error performance. Moreover, Fig. 1 shows that the error performance for $\lambda=0$ deteriorates at high SNR, because inversion of $r\left(e^{j \omega}\right)$ leads to an FFF having long effective duration. Hence, the higher is the SNR, the stronger are the detrimental effects of circular convolution. To bet- 


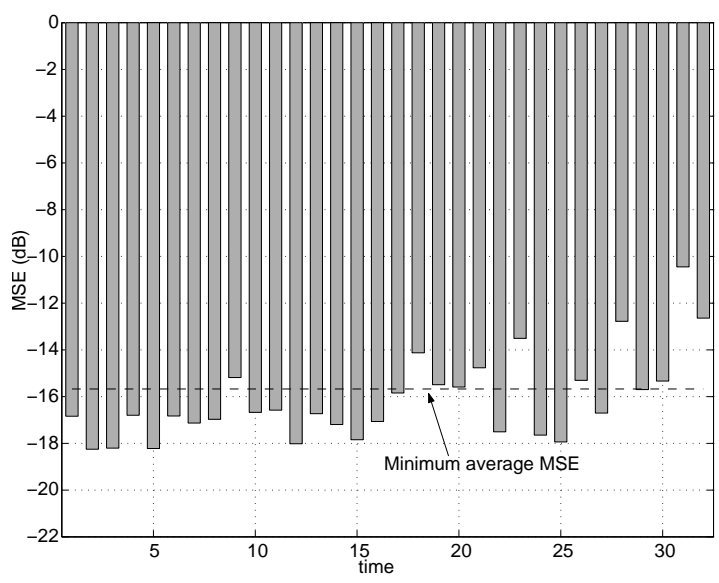

Fig. 2. Time-variant MSE in one period with $\mathrm{SNR}=$ $32.5 \mathrm{~dB}$ and $N=64$.

ter illustrate this, in figure 2 is shown the MSE versus time at $\mathrm{SNR}=32.5 \mathrm{~dB}$. The minimum average MSE is also reported in the figure. We observe that the MSE is strongly time-variant and that its greatest value occurs at time 31. From Fig. 2 it is apparent that error performance will be dominated by error events that start at time 31 .

\section{Conclusions}

Although the FFF of the MMSE-DFE is IIR, its most popular implementations are either in FIR form or in the discrete frequency domain. Frequency domain should be preferred when the duration of the FIR needed to obtain nearly optimal performance becomes too long. We have analyzed an attractive scheme of qualization where no cyclic extension is adopted and where the transfer function of the FFF is efficiently computed, but the detrimental effects of time aliasing and circular convolution may severely degrade the error performance of the scheme if no countermeasures are taken. Our main finding is that virtual noise is of great help in mitigating these detrimental effects.

\section{REFERENCES}

[1] P. Monsen, "Feedback equalization for fading dispersive channels," IEEE Trans. Inform. Theory, vol. 17, pp. 56-64, Jan. 1971.

[2] N. Al-Dhahir, J. Cioffi, "MMSE decision-feedback equalizers: finite-length results", IEEE Trans. Inform. Theory, vol. 41, pp. 961-975, July 1995.

[3] C. Belfiore, J. Park, "Decision Feedback Equalization", Proc. IEEE, vol. 67, pp. 1143-1156, Aug. 1979.

[4] D. Falconer, S. L. Ariyavisitakul, A. BenyaminSeeyar, B. Eidson, "Frequency domain equalization for single-carrier broadband wireless systems", IEEE Commun. Mag., vol. 40 , pp. 58-66, Apr. 2002.
[5] N. Benvenuto, S. Tomasin, "On the comparison between OFDM and single carrier modulation with a DFE using a frequency-domain feedforward filter", IEEE Trans. Commun., vol. 50, pp. 947-955, June 2002.

[6] S. Haykin, "Adaptive Filter Theory," Prentice Hall, 1996.

[7] E. R. Ferrara, Jr., "Fast implementation of LMS adaptive filters", IEEE Trans. Acoust., Speech Signal Processing, vol. 28, pp. 474-475, Aug. 1980.

[8] D. Mansour, A. H. Gray, Jr., "Unconstrained frequency-domain adaptive filter", IEEE Trans. Acoust., Speech Signal Processing, vol. 30, pp. 726734, Oct. 1982.

[9] A. Feuer, R. Cristi, "On the Steady State Performance of Frequency Domain LMS Algorithms", IEEE Trans. Signal Processing, vol. 41, pp. 419-423, Jan. 1993.

[10] J. C. Vanelli, N. M. Shehadeh, "Computation of bit error probability using the trapezoidal integration rule," IEEE Trans. Commun., vol. 22, pp. 331-334, March 1974.

[11] R. P. Gitlin, H. C. Meadors, S. B. Weinstein, "The tap-leakage algorithm: an algorithm for the stable operation of a digitally implemented fractionally spaced equalizer", Bell Syst. Tech. J., vol. 61, pp. 1817-1839, Oct. 1982.

[12] M. Magarini, A. Spalvieri, G. Tartara "Asymptotic analysis of stabilisation technique for the blind fractionally spaced equaliser," Electron. Lett., vol. 32, pp. 1947-1948, Oct. 1996.

[13] P. S. Bednarz, J. M. Cioffi, "Decision feedback equalization for channels with error correcting capabilities," in Proc. ICC, 1997, vol. 3, pp. 1607-1612.

[14] M. Magarini, A. Spalvieri, "MMSE decision feedback equalizer from channel estimate," in Proc. PIMRC, 2002, vol. 4, pp. 1722-1726.

[15] R. R. Anderson, G. J. Foschini, "The minimum distance for MLSE digital data systems of limited complexity," IEEE Trans. Inform. Theory, vol. 21, pp. 544-551, Sept. 1975. 